\title{
Phase-Based Manganese Enhanced MRI, a New Methodology to Enhance Brain Cytoarchitectural Contrast and Study Manganese Uptake
}

\author{
Rajika Maddage, ${ }^{1}$ José P. Marques, ${ }^{2 *}$ and Rolf Gruetter ${ }^{1,2,3}$
}

Purpose: As the magnetic susceptibility induced frequency shift increases linearly with magnetic field strength, the present work evaluates manganese as a phase imaging contrast agent and investigates the dose dependence of brain enhancement in comparison to $\mathrm{T}_{1}$-weighted imaging after intravenous administration of $\mathrm{MnCl}_{2}$.

Methods: Experiments were carried out on 12 SpragueDawley rats. $\mathrm{MnCl}_{2}$ was infused intravenously with the following doses: 25, 75, $125 \mathrm{mg} / \mathrm{kg}(\mathrm{n}=4)$. Phase, $\mathrm{T}_{1}$-weighted images and $T_{1}$ maps were acquired before and $24 \mathrm{~h}$ post $\mathrm{MnCl}_{2}$ administration at 14.1 Tesla.

Results: Manganese enhancement was manifested in phase imaging by an increase in frequency shift differences between regions rich in calcium gated channels and other tissues, together with local increase in signal to noise ratio (from the $T_{1}$ reduction). Such contrast improvement allowed a better visualization of brain cytoarchitecture. The measured $\mathrm{T}_{1}$ decrease observed across different manganese doses and in different brain regions were consistent with the increase in the contrast to noise ratio (CNR) measured by both $\mathrm{T}_{1}$-weighted and phase imaging, with the strongest variations being observed in the dentate gyrus and olfactory bulb.

Conclusion: Overall from its high sensitivity to manganese combined with excellent CNR, phase imaging is a promising alternative imaging protocol to assess manganese enhanced MRI at ultra high field. Magn Reson Med 72:1246-1256, 2014. ( 2013 Wiley Periodicals, Inc.

Key words: phase imaging; manganese enhanced MRI; MR phase contrast agent; high field MRI

\section{INTRODUCTION}

Contrast agents have been regularly used to improve the contrast between and within soft tissues and overcome the limits of the available contrast originating from differences in relaxation times $\left(\mathrm{T}_{1} / \mathrm{T}_{2}\right)$, spin mobility (diffusion, perfusion), and spin density. By affecting the longitudinal and the transverse relaxation rate of sur-

\footnotetext{
${ }^{1}$ Laboratory for Functional and Metabolic Imaging, École Polytechnique Fédérale de Lausanne, Lausanne, Switzerland.

${ }^{2}$ Department of Radiology, Université de Lausanne, Lausanne, Switzerland. ${ }^{3}$ Department of Radiology, Université de Geneve, Geneve, Switzerland.

*Correspondence to: José P. Marques, Ph.D., EPFL SB CIBM-AIT, CH F0 582 (Bâtiment $\mathrm{CH}$ ), Station 6, CH-1015 Lausanne, Switzerland. E-mail: jose.marques@epfl.ch

Received 13 May 2013; revised 16 October 2013; accepted 16 October 2013

DOI 10.1002/mrm.25037

Published online 20 November 2013 in Wiley Online Library (wileyonlinelibrary. com).
}

(c) 2013 Wiley Periodicals, Inc.

rounding water protons, paramagnetic and superparamagnetic contrast agents enhance the contrast to noise ratio (CNR) by either increasing or reducing the signal in targeted regions.

Manganese $\left(\mathrm{Mn}^{2+}\right)$ has proven to be a very interesting MR contrast agent. Being an analog to $\mathrm{Ca}^{+}$and paramagnetic (due to the five unpaired electrons on the $3 \mathrm{~d}$ electronic orbital), $\mathrm{Mn}^{2+}$ can enter voltage gated calcium channels and enhance the signal in $\mathrm{T}_{1}$-weighted images in regions of $\mathrm{Mn}^{2+}$ accumulation due to local $\mathrm{T}_{1}$ shortening. Studies using $\mathrm{Mn}^{2+}$ as a contrast agent have been limited to animal models because of its high cellular toxicity, causing heart and hepatic failure $(1,2)$, and, in the case of chronic $\mathrm{Mn}^{2+}$ exposure, leading to a form of Parkinsonism (3). In rodents, various in vivo brain studies have been performed to map neuronal connections (4), functional activity (5), and enhance the brain cytoarchitecture $(6,7)$, demonstrating to be an useful MRI marker of neuronal activity and calcium influx in tissues. As $\mathrm{Mn}^{2+}$ is not able to cross the blood brain barrier (BBB) three main approaches have been used to deliver $\mathrm{Mn}^{2+}$ to the targeted brain regions: infusion of $\mathrm{Mn}^{2+}$ followed by $\mathrm{BBB}$ disruption (8); local injection (4); systemic administration, with the enhancement being first observed in choroid plexus, cerebrospinal fluid, and only latter in tissues (9). The least invasive method, systemic administration, has enabled to depict several structures rich or with active voltage gated calcium channels such as layers in the hippocampus, cortex, and olfactory bulb $(6,7,10)$.

Over the recent years, phase imaging has gained a significant interest $(11,12)$ thanks to the increase of the magnetic fields available on clinical MR scanners. At fields above 3 Tesla, it was shown that the contrast to noise ratio (CNR) observed in phase images between gray and white matter was superior to that of conventional magnitude images, yielding additional anatomical information in the brain including the observation of subcortical contrast $(11,12)$. In rodent studies, phase imaging at high magnetic field with an in-plane resolution of $33 \mu \mathrm{m}$ was found to give anatomical details with clear depiction of cytoarchictectural features such as hippocampal fields, cortical and cerebellar layers, structures typically not easily discernible in $\mathrm{T}_{1}, \mathrm{~T}_{2}$, or $\mathrm{T}_{2}{ }^{*}$ magnitude images (13).

The observed contrast in phase imaging is due to magnetic susceptibility induced frequency shifts which increase linearly with the magnetic field strength. Even though its origin is still not clear, many mechanisms have been proposed $(11,14,15)$ with tissue bulk magnetic susceptibility playing a major role in modulating the phase shifts observed across the brain. The origin of such bulk susceptibility has been found to have 
different origins in different brain regions. In humans, the contrast observed in deep gray matter nuclei and in intracortical contrast has been attributed to local iron concentration $(16,17)$, while in rodents, the susceptibility difference responsible for gray and white matter contrast has been demonstrated to have myelin origin (18-20).

To the best of our knowledge, manganese enhanced MRI (MEMRI) has only been assessed using $\mathrm{T}_{1}$ contrast. The increasingly strong magnetic fields available potentiate stronger $\mathrm{Mn}^{2+}$ magnetic susceptibility effects, making phase imaging a natural candidate to profit from $\mathrm{Mn}^{2+}$ uptake in tissues. $\mathrm{Mn}^{2+}$ should induce a phase shift observable with phase imaging and, combined with its high sensitivity to endogenous magnetic susceptibility distribution and high CNR, could be exploited to further enhance neural architecture in animal models.

The present work was designed to investigate the above hypothesis by assessing $\mathrm{Mn}^{2+}$ potential input on phase imaging at $14.1 \mathrm{~T}$ and to determine the level of phase contrast enhancement obtained following systemic administration in comparison to the standard imaging protocol used in MEMRI, namely $\mathrm{T}_{1}$-weighted imaging. The phase shift and the contrast enhancement as a function of the $\mathrm{Mn}^{2+}$ dose were also investigated to assess its linearity and sensitivity in comparison to $\mathrm{T}_{1}$-weighted imaging. Finally, the potential of quantification of $\mathrm{Mn}^{2+}$ uptake based on phase imaging is addressed.

\section{METHODS}

\section{Animal Preparation}

Experiments were carried out in 12 male SpragueDawley rats (weighting 200-300 g). All animal experiments were conducted according to federal and local ethical guidelines and the protocols were approved by the local regulatory body.

The $\mathrm{Mn}^{2+}$ administration protocol consisted of a tail vein infusion at a rate of $1.0 \mathrm{~mL} / \mathrm{h}$ of an isotonic $\mathrm{MnCl}_{2}$ (Sigma-Aldrich, St Louis, MO) solution prepared with a concentration of $120 \mathrm{mM}$. During $\mathrm{MnCl}_{2}$ infusion, all rats were anesthetized under $2 \%$ isoflurane and their body temperature was maintained at $38 \pm 0.5^{\circ} \mathrm{C}$ by means of a heating pad. Four different doses of $\mathrm{MnCl}_{2}$ were used: 25 (4 animals), 75 (4 animals), 125 (4 animals), and $175 \mathrm{mg} / \mathrm{kg}$. The dose of $175 \mathrm{mg} / \mathrm{kg}$ was discarded due to a high mortality rate observed (90\%). After infusion, rats were returned to their cages with free access to food and water and held under a $12 \mathrm{~h} / 12 \mathrm{~h}$ light/dark regimen.

MRI was performed prior and $24 \mathrm{~h}$ post $\mathrm{MnCl}_{2}$ infusion. During the MR image acquisition, the rats were kept under anesthesia $(1.5-2 \%$ isoflurane delivered through a face mask) and were fixed using a custom made stereotaxic holder. Respiration rate was monitored using a pressure pillow placed under the rat's abdomen (respiration rate of $60 \pm 3$ breaths per minute). Body temperature was monitored using a rectal temperature probe and maintained at $38 \pm 0.5^{\circ} \mathrm{C}$ by a heated water bath.

\section{Phantom Study: $\mathrm{MnCl}_{2}$ Relaxometry at $14.1 \mathrm{~T}$}

A phantom containing seven cylindrical tubes with different $\mathrm{Mn}^{2+}$ concentration (from $0 \mu \mathrm{M}$ to $200 \mu \mathrm{M}$ dissolved in distilled water) was produced. From the $\mathrm{T}_{1}(1 /$ $\mathrm{R}_{1}$ ) and frequency shift maps measured across the $\mathrm{Mn}^{2+}$ concentrations, it was possible to compute the relaxivity parameters $r_{1}$ and magnetic susceptibility $\chi\left(\left[\mathrm{Mn}^{2+}\right]\right)$. (Details of the imaging and processing protocols will be given in the MRI and Phantom Data sections respectively).

\section{MRI}

All scans were performed on a $14.1 \mathrm{~T} / 26 \mathrm{~cm}$ horizontal bore magnet (Varian/Magnex Scientific). The $\mathrm{MnCl}_{2}$ relaxometry protocol (performed on the phantom described on the previous paragraph) was acquired using a high pass birdcage coil with an inner diameter of 46 $\mathrm{mm}$ and length of $30 \mathrm{~mm}$. In vivo rat brain images were acquired using a home built quadrature surface coil as radiofrequency $(\mathrm{RF})$ transceiver with two geometrically decoupled $21 \mathrm{~mm}$ loops resonating at $600 \mathrm{MHz}$. To take full advantage of the high signal to noise ratio (SNR), the surface coil was carefully and systematically positioned over the brain regions under investigation, regions known to be rich/or with active voltage gated calcium channels that are susceptible to $\mathrm{Mn}^{2+}$ uptake $(6,7)$ : olfactory bulb (OB) including the olfactory nerve layer (ONL), glomerular layer (GL) and mitral cell layer (ML); the hippocampus (HC) including the CA formation of the hippocampus and the dentate gyrus. To take advantage of using thick slices (compared with the in-plane resolution) and to further improve the SNR without significantly increasing partial volume effects, both the slices covering the hippocampus and the olfactory bulb were positioned coronally.

Magnetic field homogeneity was adjusted using FASTMAP (21) in a large volume located in the different regions. Water spectra linewidth of $21-26 \mathrm{~Hz}$ were obtained over a typical voxel size of $7 \times 10 \times 6 \mathrm{~mm}^{3}$ in cortex/hippocampus regions and $2 \times 2 \times 4 \mathrm{~mm}^{3}$ in the OB. The complete acquisition protocol described in the following paragraph was applied before and $24 \mathrm{~h}$ post $\mathrm{Mn}^{2+}$ systemic administration.

\section{$\mathrm{T}_{1}$ Mapping}

$\mathrm{T}_{1}$ mapping was performed using a multi-slice multishot inversion-recovery Look-Locker Segmented Echo Planar Imaging sequence $(22,23)$. A slice-selective $180^{\circ}$ adiabatic pulse (hyperbolic secant, hs8) was used to invert the magnetization followed by low flip angle slice-selective pulses $\left(\mathrm{FA}=20^{\circ}\right)$ that sampled the inversion recovery curve in 20 points with inversion times from $50 \mathrm{~ms}$ to $4050 \mathrm{~ms}$ (with an inter-excitation delay of $200 \mathrm{~ms}$ ). The repetition time (TR) was set to $24.5 \mathrm{~s}$, sufficient for the full recovery of the longitudinal magnetization allowing 6 slices to be acquired per TR while the interexcitation delay was chosen to guarantee the accuracy of $\mathrm{T}_{1}$ values down to $400 \mathrm{~ms}(22,23)$. The echo time (TE) of the EPI readout was kept short $(\mathrm{TE}=5.7 \mathrm{~ms}$ ) to avoid through slice signal dephasing. K-space was 
acquired in a segmented manner (16 shots) to reduce geometrical distortions due to magnetic susceptibility differences (tissue/air). Other imaging parameters: field of view $(\mathrm{FOV})=22 * 22 \mathrm{~mm}^{2}$, resolution $=172 * 172 * 800$ $\mu \mathrm{m}^{3}$, number of slices $=6$ and total acquisition time $=$ 14 min. For Nyquist ghost correction, a readout polarity reversal scheme was used (24).

\section{$\mathrm{T}_{1}$-Weighted and Phase Images}

$\mathrm{T}_{1}$-weighted and phase images were acquired using a gradient echo multi-slice sequence (GEMS) with respiration gating to reduce respiration induced artifacts which suggested that all images were acquired with a TR 1s. The small and slow variations in respiration rate could only generate a maximum signal variation (due to TR fluctuations) of $\pm 4 \%$, which was considered too small to require any extra corrections.

For phase images, the flip angle was optimized to maximize the signal intensity in the specific regions of interest. The echo time (16 ms) was chosen to approximately match the gray matter $\mathrm{T}_{2}{ }^{*}$ and, hence, optimize phase contrast (13). The acquisition bandwidth was chosen to maximize signal without introducing effective resolution reduction due to $\mathrm{T}_{2}{ }^{*}$ decay during the readout (tacq $=$ $15.9 \mathrm{~ms})$.

For $\mathrm{T}_{1}$-weighted images, echo time was reduced (5 ms) and acquisition bandwidth was increased to emphasize the $\mathrm{T}_{1}$ contrast while maintaining sufficient SNR. The flip angle was chosen to be close to $90^{\circ}$ to enhance the $\mathrm{T}_{1}$ contrast in the regions where contrast enhancement was expected, while avoiding undesirable inversions in the regions closest to the coil.

Summary of acquisition parameters: TR 1s (respiration gating), $\mathrm{TE}=5 \mathrm{~ms}\left(\mathrm{~T}_{1}\right.$-weighted) $/ 16 \mathrm{~ms}$ (Phase), FA $=90^{\circ}\left(\mathrm{T}_{1}\right.$-weighted $) / 50^{\circ}$ (Phase), number of slices $=20$ with $0.2-\mathrm{mm}$ gap to avoid cross excitation between slices. The image resolution in the different regions was $66 * 66 * 400 \mu \mathrm{m}^{3}$ (Cortex /hippocampus), 58*58*400 $\mathrm{\mu m}^{3}$ (OB) with a total acquisition time of $8 \mathrm{~min}$ (Cortex/hippocampus, $\mathrm{OB}$ ).

\section{Frequency Shift Maps}

To establish the magnetic susceptibility of $\mathrm{Mn}^{2+}$ in the phantom, frequency shift maps were acquired using a multi-gradient echo sequence. Fifteen gradient echo images ( 4.5 to $60.5 \mathrm{~ms}$ ) were obtained with the following parameters: $\mathrm{TR}=900 \mathrm{~ms}, \mathrm{FA}=40^{\circ}, \mathrm{FOV}=30^{*} 30 \mathrm{~mm}^{2}$, resolution $=234 * 234 * 800 \mu \mathrm{m}^{3}$.

\section{Data Processing}

$\mathrm{T}_{1}$ maps were calculated pixel by pixed using a NelderMead fitting algorithm (25). Due to the coil setup (surface coil) a three-parameter fit $\left(\mathrm{T}_{1}, \mathrm{M}_{0}\right.$ and flip angle $\left.\alpha\right)$ was done to take into account the transmit field inhomogeneity. Steady state signal was considered in the algorithm to remove any bias from possible magnetization not fully recovered at the end of the TR. Frequency maps were established from a linear fit to the phase change over the 15 echoes. Phase images were unwrapped (26) and the background field contributions originating from imperfect shimming, air tissue interfaces were filtered using the SHARP algorithm (17). This methodology does not remove entirely phase variations related to the transmitting and receiving $B_{1}$ field which can be removed by applying a high pass filter with a wide Gaussian width.

\section{Phantom Data}

Regions of interest (ROIs) were placed within each $\mathrm{MnCl}_{2}$ tube. The relaxation rate $\mathrm{R}_{1}$ and frequency values were measured from the respective $\mathrm{T}_{1}$ and frequency maps for each ROI. The different values were then plotted as a function of $\left[\mathrm{Mn}^{2+}\right]$ and a linear regression was performed to find the longitudinal relaxivity, $r_{1}$, of $\mathrm{Mn}^{2+}$

$$
R_{1}\left(\left[M n^{2+}\right]\right)=R_{1}(0)+r_{1} \cdot\left[M n^{2+}\right] .
$$

For the frequency map, the outside compartment $\left(\left[\mathrm{Mn}^{2+}\right]=0\right)$ was used as a reference. A frequency shift per millimolar, $\delta f_{p m M}$, was calculated

$$
\Delta f\left(\left[M n^{2+}\right]\right)=\delta f_{p m M} \cdot\left[M n^{2+}\right] .
$$

Subsequently, the magnetic susceptibility per millimolar was calculated using the following expression $\delta f_{p m M}=$ $-1 / 3^{*} \gamma B_{0} \chi_{\text {pmM }}$ (27), where $\gamma$ is the gyromagnetic constant ratio, which represents the relationship between the frequency shift difference and the susceptibility difference for an infinite cylinder aligned along the main magnetic field.

\section{In Vivo Data}

The changes in $\mathrm{T}_{1}$ due to $\mathrm{Mn}^{2+}$ as a function of the different doses were calculated by selecting ROIs in the cortex, hippocampus, corpus callosum (within the same slice) and in different fields of the OB (Fig. 4). Although manganese can be transported by means of the axons (28), its enrichment in white matter is limited as it mainly accumulates in synaptic regions of gray matter (29). To measure the contrast enhancement throughout the $\mathrm{Mn}^{2+}$ doses, the CNR was calculated as the signal difference between the tissue of interest and a region devoid of $\mathrm{Mn}^{2+}$ enhancement (WM) divided by the standard deviation of the noise $(\sigma)$. The $\mathrm{T}_{1}$-weighted contrast of the cortex and dentate gyrus of the hippocampus were established in respect to the corpus callosum whereas the contrast of the OB was established by subtracting the signal from the anterior commissure, intrabulbar part (aci) (Fig. 4).

Likewise, phase enrichment $\left(\Delta f_{G M-W M}\right)$ and phase CNR were established considering ROI's in the same slice and the latter was calculated using the following equation:

$$
C N R_{\text {phase }}=\text { Signal }_{a v} \cdot 2 \pi \cdot T E \cdot\left|f_{G M}-f_{W M}\right| / \sigma .
$$

With Signal $_{a V}=\left(\right.$ Signal $_{G M}+$ Signal $\left._{W M}\right) / 2$, TE the echo time used, $\mathrm{f}_{\mathrm{GM}}$ and $\mathrm{f}_{\mathrm{WM}}$ the frequency shift of gray matter and white matter, respectively, and $\sigma$ the standard deviation of the background noise measured outside the brain within artifact free regions (11). 

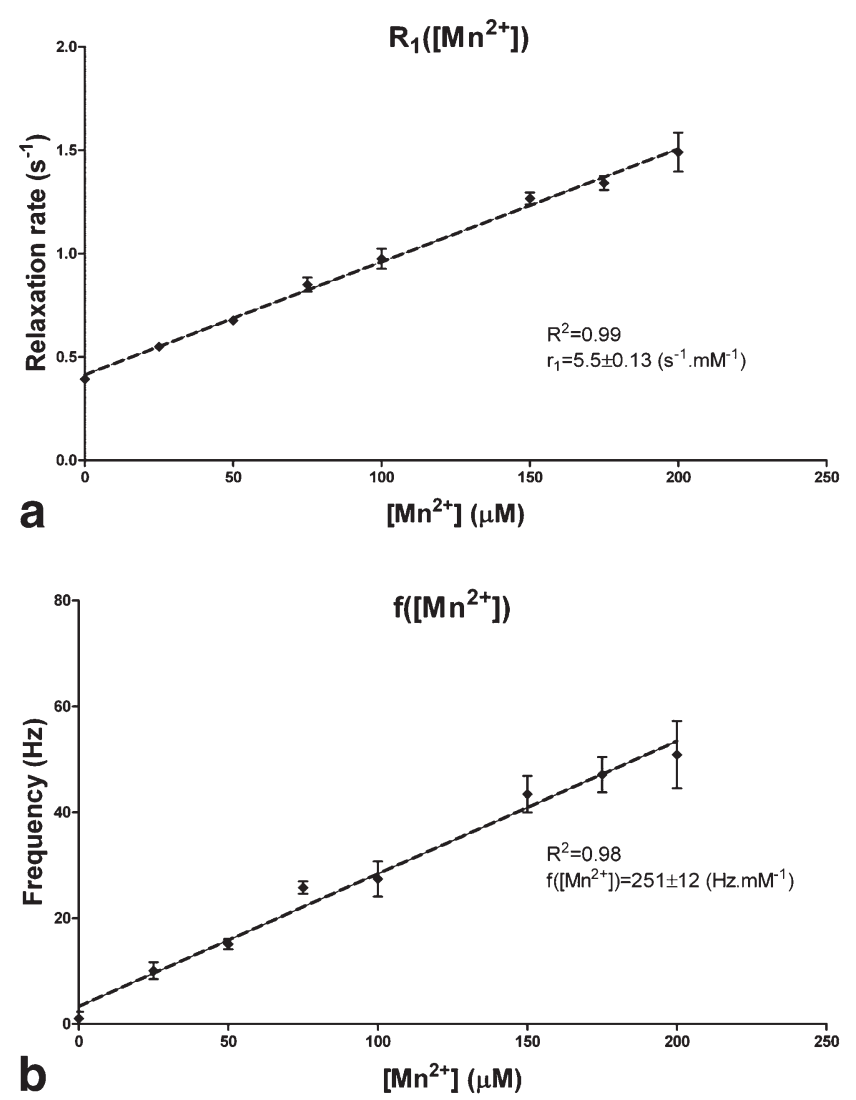

FIG. 1. In vitro relaxivity measurements of $\mathrm{Mn}^{2+}$ at $14.1 \mathrm{~T}$. a: Longitudinal relaxation rate $R_{1}$. b: Frequency shift plotted as function of $\left[\mathrm{Mn}^{2+}\right]$. Error bars represent standard deviations over the regions of interest. Dashed line represents the linear regression fit to the data points.

\section{RESULTS}

Relaxivity $\mathrm{r}_{1}$ and $\delta f_{p m M}$ of $\mathrm{Mn}^{2+}$ at $14.1 \mathrm{~T}$

To determine the relaxivity parameters of $\mathrm{Mn}^{2+}$ at $14.1 \mathrm{~T}$, the relaxation rates, $R_{1}$, and frequency shifts, $\Delta f$, were measured using a phantom containing seven cylindrical tubes with $\mathrm{Mn}^{2+}$ concentrations ranging from 0 to $200 \mu \mathrm{M}$, (Fig. 1). Both quantities showed the expected linear relationship with $\mathrm{Mn}^{2+}$ concentration $\left(\mathrm{R}^{2}>0.98\right)$. The measured longitudinal relaxivity, $\mathrm{r}_{1}$, was established at $5.5 \pm$ $0.1 \mathrm{~s}^{-1} \cdot \mathrm{mM}^{-1}$ (Fig. 1a). The frequency shift as a function of $\mathrm{Mn}^{2+} \delta f_{p m M}$ was measured at $251 \pm 12 \mathrm{~Hz} \cdot \mathrm{mM}^{-1}$ (Fig. 1b) resulting in a susceptibility of $\mathrm{Mn}^{2+}$ of $-1.25 \pm 0.06$ ppm. $\mathrm{mM}^{-1}$.The apparent transverse relaxivity $\mathrm{r}_{2}{ }^{*}$ was also established at $234 \pm 8 \mathrm{~s}^{-1} \cdot \mathrm{mM}^{-1}$ (data not shown).

Induced Phase and $\mathrm{T}_{1}$ Changes 24h Post Systemic $\mathrm{Mn}^{2+}$ Administration

\section{$T_{1}$-weighted imaging}

To qualitatively determine the effect of systemic $\mathrm{Mn}^{2+}$ administration on $\mathrm{T}_{1}$ contrast, $\mathrm{T}_{1}$-weighted images of the olfactory bulb, cortex, and hippocampus acquired before and $24 \mathrm{~h}$ after a $125 \mathrm{mg} / \mathrm{kg}$ systemic $\mathrm{Mn}^{2+}$ administration were compared (Fig. 2).
Before $\mathrm{Mn}^{2+}$ administration, tissue structures in the OB, cortex, and hippocampus regions were poorly discernible despite the high in-plane resolution of $58 \mu \mathrm{m}$ (Fig. 2).

Twenty-four hours after $\mathrm{Mn}^{2+}$ systemic administration, an overall increase in SNR was observed in all regions (OB: $+31 \%$; cortex $+38 \%$; hippocampus: $+66 \%$ ) followed by a significant increase in tissue contrast in manganeseenhanced structures. In the OB, several anatomic features were enhanced and well depicted such as the external plexiform (EPL), internal plexiform (IPL), the mitral cell layer (ML), granular cell layer (GrL), and olfactory nerve layer (ONL. Subregional anatomic structures of the hippocampus were also well visualized with clear depiction of CA1, CA2, CA3, DG of the hippocampus (black arrows Fig. 2).

\section{Phase imaging}

To qualitatively determine the effect of $\mathrm{Mn}^{2+}$ on phase contrast, phase images acquired before and $24 \mathrm{~h}$ after a $125 \mathrm{mg} / \mathrm{kg} \mathrm{Mn}^{2+}$ administration, in the same slices/ regions of interest as the $\mathrm{T}_{1}$-weighted images, were compared (Fig. 3).

Before $\mathrm{Mn}^{2+}$ administration, the high contrast to noise ratio (CNR) given by the phase image already enabled to depict several structures of the olfactory bulb such as the external plexiform (EPL) and internal plexiform (IPL), granular cell layer (GrL), and olfactory nerve layer (ONL) (Fig. 3). Twenty-four hours following $\mathrm{Mn}^{2+}$ administration, $\mathrm{Mn}^{2+}$ accumulation resulted in an increase of CNR in all structures rich or with active voltage gated calcium channels, further enhancing the cytoarchitectural features (black arrows Fig. 3) which were already depicted beforehand with a clear emphasis of the mitral cell layer (ML) and the glomerular layer (GL).

Likewise, in the cortex-hippocampus region and before $\mathrm{Mn}^{2+}$ administration, phase imaging yielded substantial anatomical contrast allowing the identification of hippocampus structures, gray matter cortical layer (Fig. 3) and white matter structures such as the corpus callosum, the internal capsule, and the fornix (as expected from previous studies). A phase shift and CNR increase mainly in the dentate gyrus, CA of the hippocampus and cortical layer (black arrows Fig. 3) was likewise observed twenty four hours following $\mathrm{Mn}^{2+}$ administration further enhancing the cytoarchitectural features which were already depicted beforehand.

\section{Dose Dependence of $T_{1}$ and Phase Contrast following $\mathrm{Mn}^{2+}$ Systemic Administration}

To define the effect of the dose of $\mathrm{Mn}^{2+}$ administration on both $\mathrm{T}_{1}$ and phase images, the $\mathrm{Mn}^{2+}$ dose was varied from $0,25,75$, and $125 \mathrm{mg} / \mathrm{kg}$. Figure 4 shows the corresponding $\mathrm{T}_{1}$ map, phase and $\mathrm{T}_{1}$-weighted images acquired in the cortex-hippocampus and olfactory bulb regions with $\mathrm{Mn}^{2+}$ doses ranging from 0 to $125 \mathrm{mg} / \mathrm{kg}$.

At the lowest dose for both phase and $\mathrm{T}_{1}$-weighted images, enhancement of the CA1, CA2, CA3, and DG of the hippocampus (Fig. 4a) and external, internal plexiform, mitral cell, olfactory nerve, and granular cell layers (Fig. 4b) were already visible. In comparison to the cortexhippocampus region, the contrast enhancement in the olfactory bulb was more pronounced for both imaging methods. 


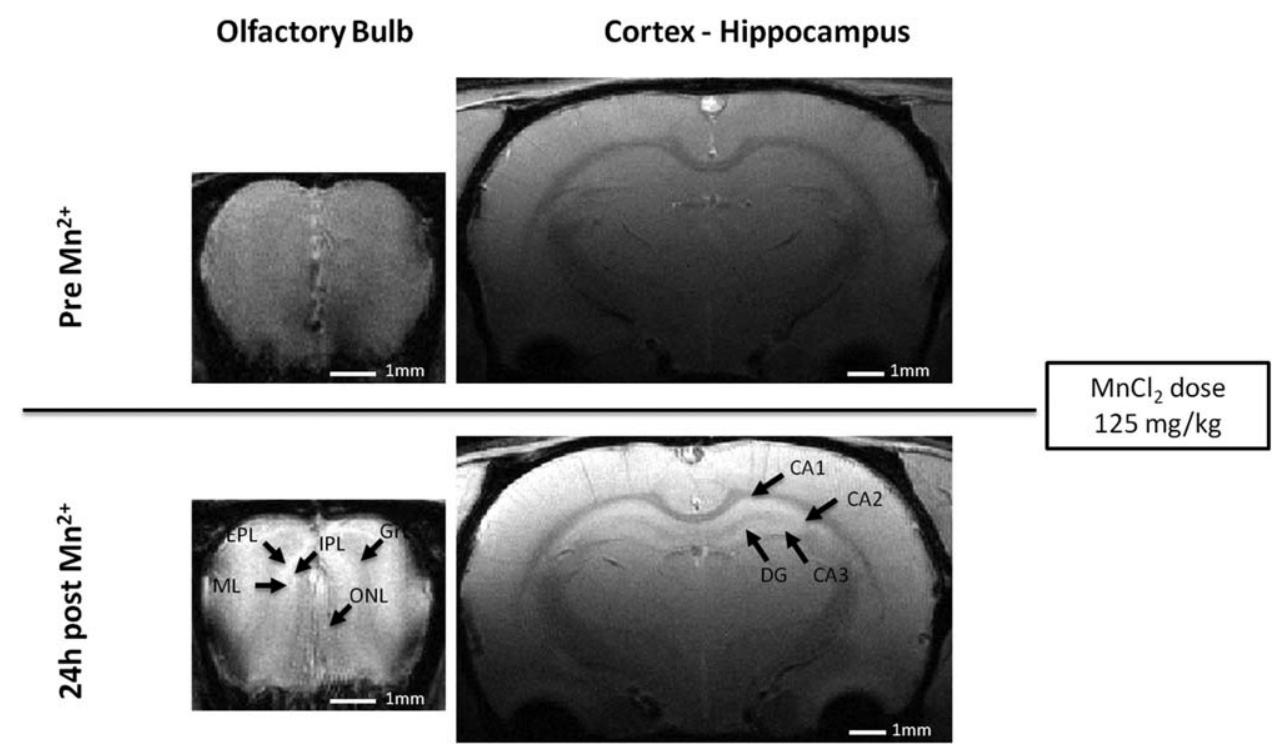

FIG. 2. Axial $T_{1}$-weighted images of the Olfactory Bulb, Cortex and Hippocampus before and $24 \mathrm{~h}$ post $\mathrm{MnCl}_{2}$ i.v. systemic administration (dose of $125 \mathrm{mg}$ / $\mathrm{kg}$ ). Pronounced signal and contrast enhancement of the brain cytoarchitecture with clear depiction of the CA1, CA3, DG of the hippocampus and olfactory bulb layers (black arrows). EPL: external plexiform; IPL: internal plexiform; ML: mitral cell layer; ONL: olfactory bulb nerve layer; GrL: granular cell layer; DG: dentate gyrus.

Such enhancement progressed with the increasing doses of $\mathrm{Mn}^{2+}$, offering a better delineation of the cytoarchitectural features cited above in both imaging methods. These observations were supported by the observed $\mathrm{T}_{1}$ decrease in the corresponding $\mathrm{T}_{1}$ maps. Quantification of the $R_{1}$ increase is shown in Figure 5 a as the evolution of the relaxation rate $\Delta R_{1}$ in regions of $\mathrm{Mn}^{2+}$ enhancement and in white matter as a function of the $\mathrm{Mn}^{2+}$ dose. (Preinfused R1: Cortex: $0.42 \mathrm{~s}^{-1} \pm 0.01$; Dentate Gyrus: $0.46 \mathrm{~s}^{-1} \pm$ 0.01; Olfactory Bulb: $0.46 \mathrm{~s}^{-1} \pm$ 0.03; Corpus callosum: $\left.0.51 \mathrm{~s}^{-1} \pm 0.02\right) . \Delta \mathrm{R}_{1}$ was calculated for a specific ROI as the difference in relaxation rate between a given $\mathrm{Mn}^{2+}$ dose and preinfusion control. From $0 \mathrm{mg} / \mathrm{kg}$ to $125 \mathrm{mg} / \mathrm{kg}, \Delta \mathrm{R}_{1} \mathrm{~s}$ in all regions increased, with the dentate gyrus showing a greatest increase indicating a higher $\mathrm{Mn}^{2+}$ uptake followed by the olfactory bulb and cortex. $\Delta \mathrm{R}_{1}$ of the corpus callosum showed minimal increase throughout the $\mathrm{Mn}^{2+}$ doses $\left(+0.02 \mathrm{~s}^{-1} \pm 0.03\right.$ from 0 to $\left.125 \mathrm{mg} / \mathrm{kg}\right)$.

Figure $5 \mathrm{~b}$ shows the evolution of the frequency shift difference $\Delta \mathrm{f}$ as a function of $\mathrm{Mn}^{2+}$ dose measured in the same ROIs as in Figure 5a. $\Delta \mathrm{f}$ was calculated in the same manner as $\Delta \mathrm{R}_{1}$. However, as the frequency is not absolute (due to background field removal), $\Delta \mathrm{f}$ was

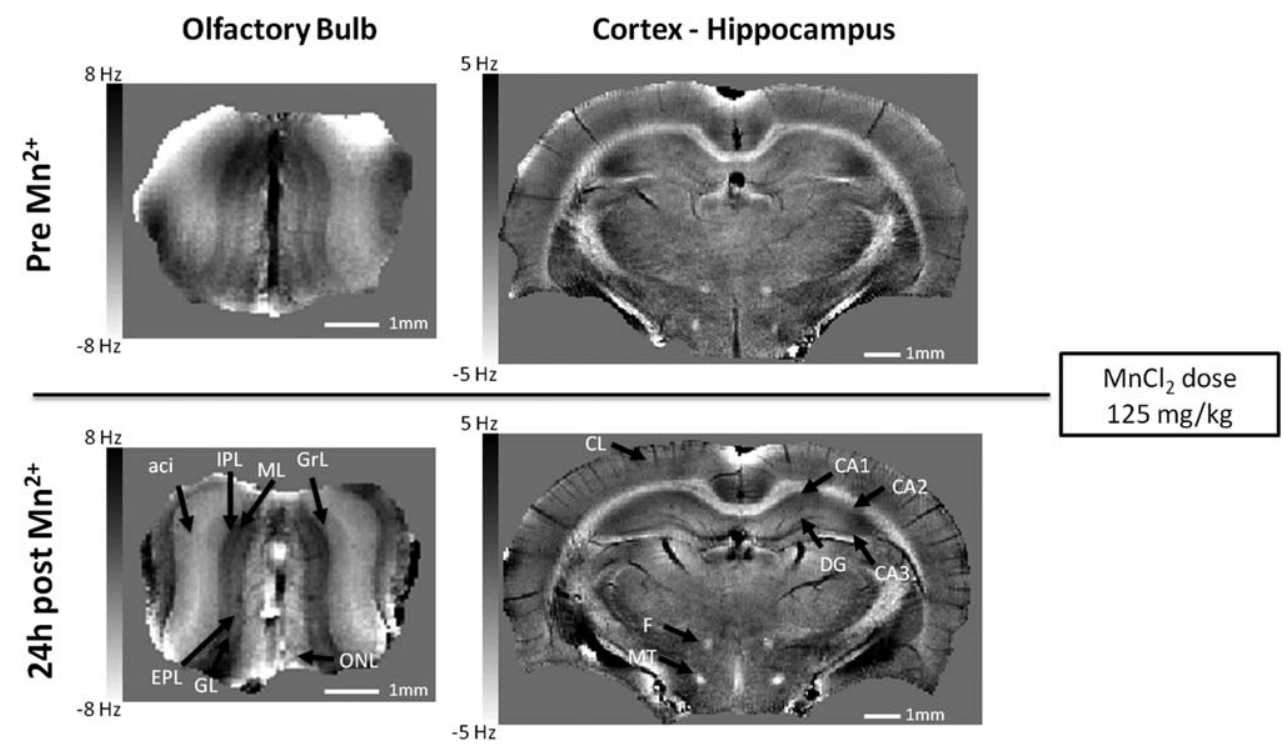

FIG. 3. Axial gradient echo phases images of the olfactory bulb, cortex and hippocampus before and $24 \mathrm{~h}$ post $\mathrm{MnCl}_{2}$ i.v. systemic administration (dose of $125 \mathrm{mg} / \mathrm{kg}$ ). Pre $\mathrm{Mn}^{2+}$ phase images with in plane resolution of $58 \mu \mathrm{m} / \mathrm{OB}$ and $66 \mu \mathrm{m} /$ Cortex-hippocampus region acquired in 8 min show good CNR between GM and WM structures enabling to already depict several tissue structures only seen in $\mathrm{T}_{1}$-weighted images following $\mathrm{Mn}^{2+}$ systemic administration. Twenty-four hours post $\mathrm{Mn}^{2+}$ infusion, $\mathrm{Mn}^{2+}$ accumulation in regions rich or with active calcium channels resulted in a positive phase shift from the induced magnetic susceptibility and an increase in phase CNR. EPL: external plexiform; IPL: internal plexiform; ML: mitral cell layer; ONL: olfactory bulb nerve layer; GrL: granular cell layer; DG: dentate gyrus; F: fornix; MT: mammillothalamic tract. 

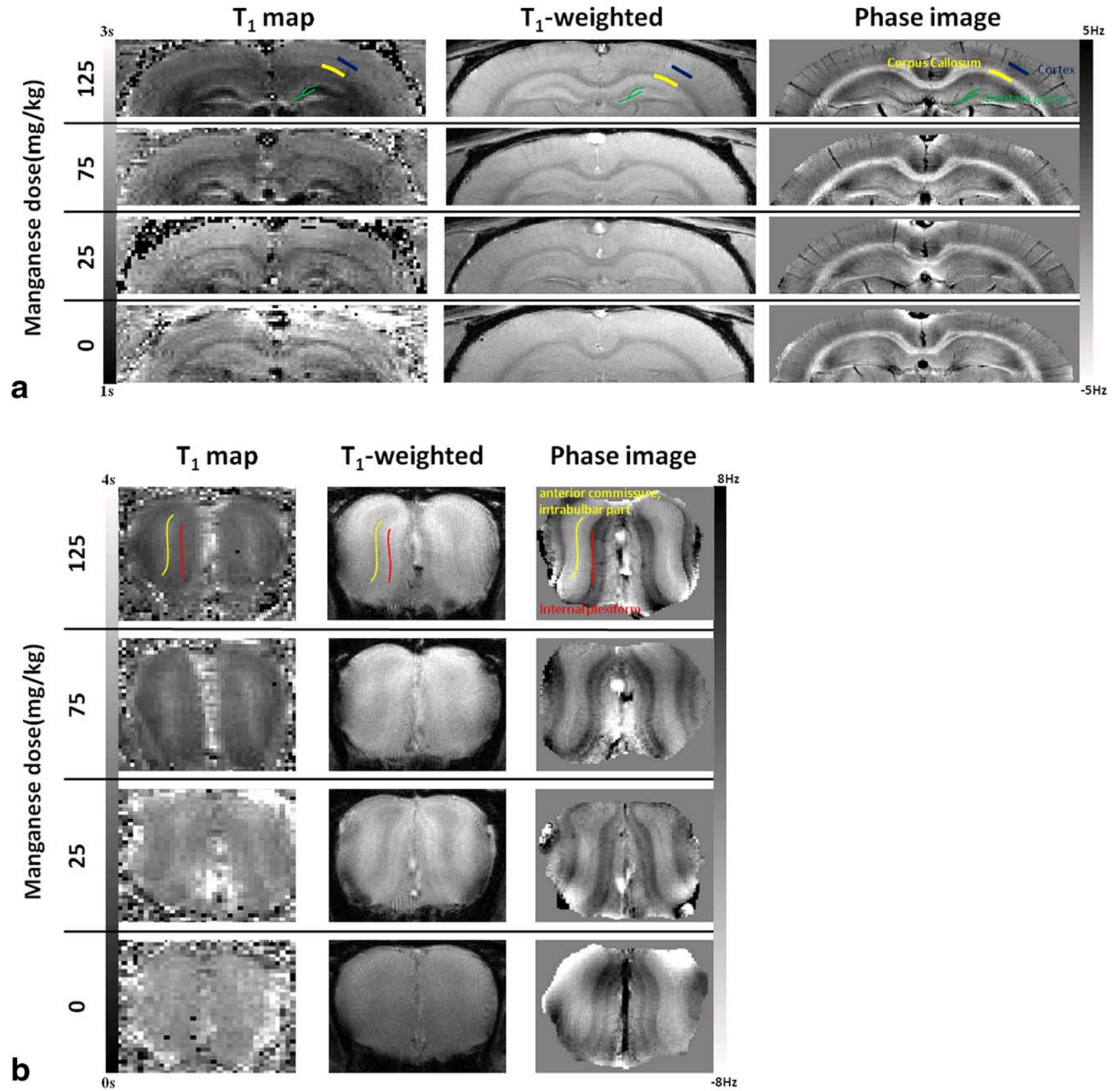

FIG. 4. Dose dependence of MEMRI contrast in cortex-hippocampus region (a) and OB (b) observed in corresponding $T_{1}$ map, $\mathrm{T}_{1}$-weighted and phase images taken from the same animal. From the lowest dose of $25 \mathrm{mg} / \mathrm{kg}$, brain cytoarchitecture is enhanced in both $\mathrm{T}_{1}$-weighted and phase images with improved CNR and SNR which increased with respect to $\mathrm{Mn}^{2+}$ dose offering clear delineation of several subregional structures in the hippocampus and olfactory bulb. Signal intensity of $\mathrm{T}_{1}$-weighted images has been normalized by the standard deviation of the background noise calculated from an artifact free region. Regions of interest drawn for quantification analysis are shown in the $125 \mathrm{mg} / \mathrm{kg}$ images for (a) and (b). [Color figure can be viewed in the online issue, which is available at wileyonlinelibrary.com.]

established by subtracting respectively the frequency value of the white matter in both $\mathrm{Mn}^{2+}$ and preinfusion control data (corpus callosum for the cortex and dentate gyrus; anterior commissure intrabulbar part for the olfactory bulb-yellow regions in Figs. 3 and 4). Again, a monotonic increase of the frequency difference as a function of $\mathrm{Mn}^{2+}$ uptake is observed. The smallest increase of $\Delta \mathrm{f}$ was again observed in the cortex. However, in opposition to $\Delta \mathrm{R}_{1}$, the olfactory bulb showed the greatest $\Delta$ f increase.

\section{$\mathrm{T}_{1}$-Weighted and Phase CNR Comparison}

To compare the sensitivity of phase imaging and $\mathrm{T}_{1^{-}}$weighted images at $14.1 \mathrm{~T}$, the dose dependence of the CNR was compared for both imaging methods and in the same regions of interest as described in the "Data Processing” paragraph. CNR for both imaging methods was established by subtracting the signal of neighboring white matter for each region and for each given $\mathrm{Mn}^{2+}$ dose. Figure 6 and Table 1 show that with increasing 

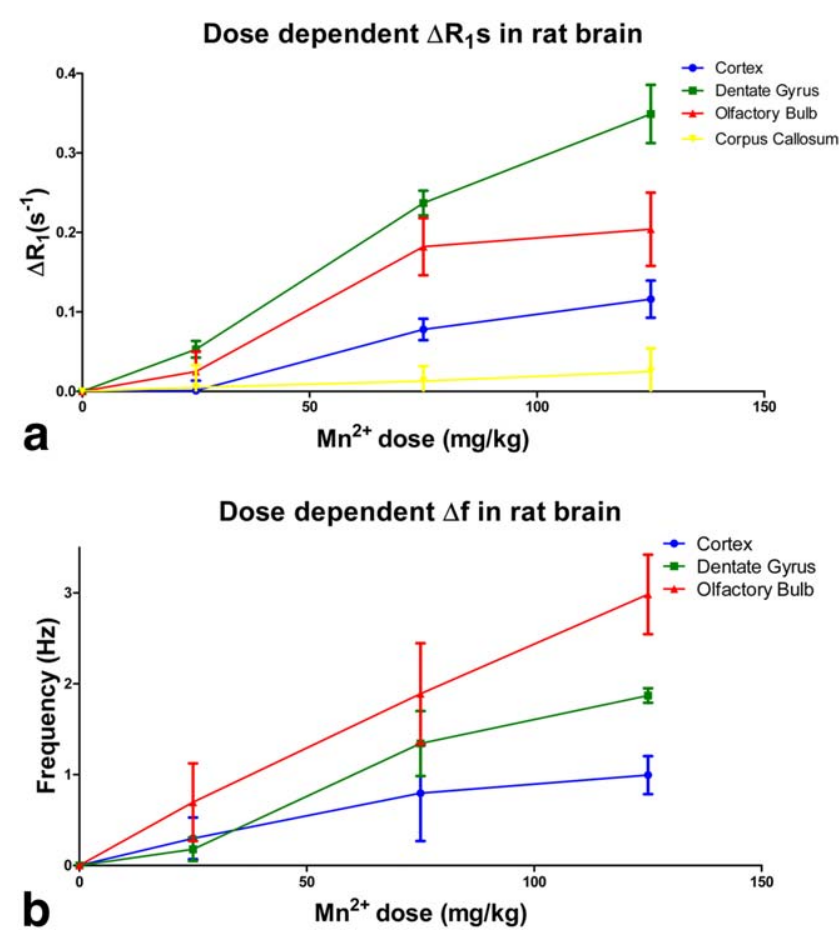

FIG. 5. $\Delta R_{1}$ (a) and $\Delta f(b)$ evolution, function of manganese dose across different brain regions. For a specific region of interest (ROI), $\Delta \mathrm{R}_{1}$ and $\Delta \mathrm{f}$ were established as follows: $\Delta \mathrm{R}_{1}\left(\mathrm{Mn}^{2+}{ }_{\text {dose }}\right)=$ $R_{1}\left(M n^{2+}\right.$ dose)- $R_{1}(0)$ with $R_{1}(0)$, preinfusion control $R_{1}$ value for corresponding brain region. Likewise, $\Delta \mathrm{f}\left(\mathrm{Mn}^{2+}{ }_{\text {dose }}\right)=\left(\mathrm{f}\left(\mathrm{Mn}^{2+} \text { dose }\right)^{-}\right.$ $f_{W M}\left(M n^{2+}\right.$ dose $\left.)\right)-\left(f(0)-f_{W M}(0)\right) . \Delta f$ in the dentate gyrus and cortex was measured by subtracting the WM frequency of the corpus callosum in both $\mathrm{Mn}^{2+}$ and preinfusion control data. $\Delta f$ of the olfactory bulb was measured by subtracting the WM frequency of the anterior commissure, intrabulbar part. From $0 \mathrm{mg} / \mathrm{kg}$ to 125 $\mathrm{mg} / \mathrm{kg}$, the dentate gyrus showed the highest increase indicating a higher $\mathrm{Mn}^{2+}$ uptake followed by the olfactory bulb and cortex. Likewise, a monotonic increase of the frequency difference as a function of $\mathrm{Mn}^{2+}$ uptake was observed in all regions. The smallest increase of $\Delta f$ was again observed in the cortex. However, in opposition to $\Delta R_{1}$, the olfactory bulb showed the highest $\Delta f$ increase. [Color figure can be viewed in the online issue, which is available at wileyonlinelibrary.com.]

$\mathrm{Mn}^{2+}$ dose, both phase and $\mathrm{T}_{1}$-weighted CNR increased progressively in all regions of interest. Globally, phase contrast outperformed our $\mathrm{T}_{1}$-weighted imaging protocol showing always greater contrast. Also the CNR increase was greater in 7 of the 9 regions studied for the phase contrast when compared with the $\mathrm{T}_{1}$-weighted contrast. In terms of relative increase of the CNR (relative to the initial contrast, see values inside the brackets on Table 1) between the control region and the regions with active $\mathrm{Mn}^{2+}$ uptake, $\mathrm{T}_{1}$-weighted images showed superior fractional CNR increase in 6 of the 9 regions studied.

\section{DISCUSSION}

The study reports for the first time the longitudinal relaxivity parameters $\left(r_{1}\right)$ and the magnetic susceptibility $\chi_{\mathrm{pmM}}$ of $\mathrm{Mn}^{2+}$ at $14.1 \mathrm{~T}$. Magnetic susceptibility values demonstrate that $\mathrm{Mn}^{2+}$ enhancement could be explored in phase imaging (Fig. 1). The relaxivity of $\mathrm{Mn}^{2+}$ estab- lished from the measurements of $\mathrm{R}_{1}$ to varying $\mathrm{Mn}^{2+}$ concentrations was in good agreement with $r_{1}$ reported at $1.5 \mathrm{~T}(29)$ and at $11.7 \mathrm{~T}(30)$.

These in vitro observations were confirmed in vivo with enhancement of regions rich in voltage gated calcium channels in both phase and $\mathrm{T}_{1}$-weighted images 24h following $\mathrm{Mn}^{2+}$ administration (as seen Figs. 3 and 2 , respectively). The results from the $\mathrm{T}_{1}$-weighted images are consistent with previous MEMRI studies $(6,7,10)$. $\mathrm{Mn}^{2+}$ enhancement was manifested in $\mathrm{T}_{1}$-weighted imaging by a signal increase due to the reduction in $\mathrm{T}_{1}$. In phase imaging, $\mathrm{Mn}^{2+}$ uptake not only resulted in increased frequency shift differences (penultimate term of Eq. [3]) but also in increased SNR due to the reduction in $\mathrm{T}_{1}$ (first term of Eq. [3]). $\mathrm{Mn}^{2+}$ uptake also introduces a signal attenuation due to $\mathrm{T}_{2}{ }^{*}$ decay which could hamper, at high magnetic field, the assessment of MEMRI contrast by phase imaging for high $\mathrm{Mn}^{2+}$ concentrations and long echo times. However, for the typically $\mathrm{Mn}^{2+}$ concentrations found in vivo, it should be noted that this is not a concern as the phase CNR continues to increase (see Appendix 1). The gain in phase contrast associated with $\mathrm{T}_{1}$ reduction and increased frequency shift make phase imaging, and potentially susceptibility mapping, a promising tool in the field of MEMRI.

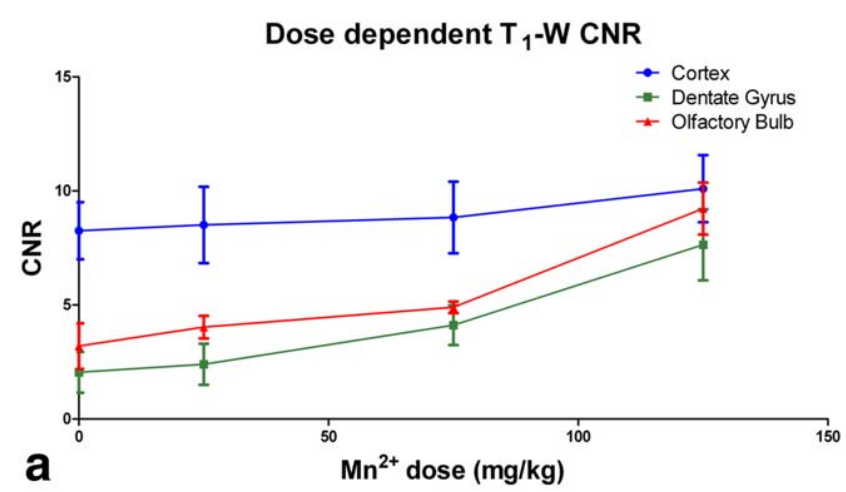

\section{Dose dependent phase CNR}

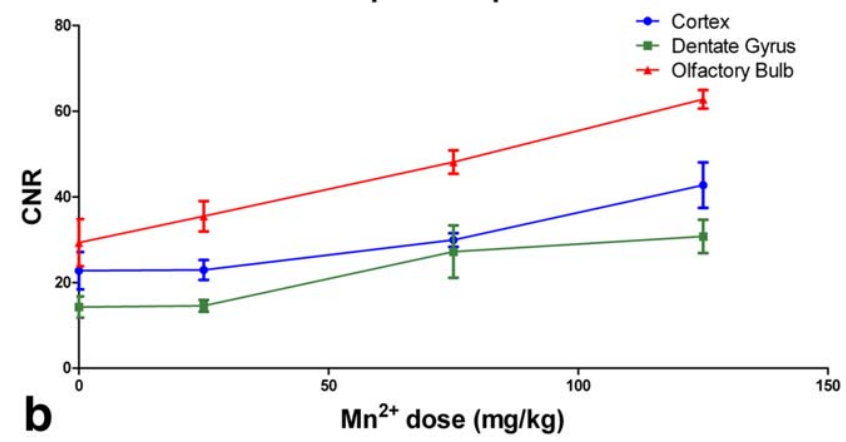

FIG. 6. Plots of the contrast to noise ratio of $\mathrm{T}_{1}$-weighted (a) and phase imaging (b) as a function of the manganese dose for different brain regions (cortex, dentate gyrus, and olfactory bulb). At control level and for all regions, phase CNR was significantly higher than $\mathrm{T}_{1}$-weighted CNR. From 0 to $125 \mathrm{mg} / \mathrm{kg}$, both phase and $T_{1}$-weighted $C N R$ increased progressively in all regions of interest. The cortex exhibited the slowest CNR increase in both imaging methods while the dentate gyrus and olfactory bulb showed the highest. [Color figure can be viewed in the online issue, which is available at wileyonlinelibrary.com.] 
Table 1

$\mathrm{Mn}^{2+}$ Dose Dependence $\mathrm{T}_{1}$-Weighted and Phase CNR Values in Different Brain Regions in Respect to Neighboring White Matter Regions ${ }^{a}$

\begin{tabular}{|c|c|c|c|c|c|c|}
\hline \multirow{2}{*}{$\begin{array}{l}\mathrm{Mn}^{2+} \\
\text { dose } \\
(\mathrm{mg} / \mathrm{kg})\end{array}$} & \multicolumn{2}{|c|}{ Cortex } & \multicolumn{2}{|c|}{ Dentate gyrus } & \multicolumn{2}{|c|}{ Olfactory bulb } \\
\hline & T1W CNR & Phase CNR & T1W CNR & Phase CNR & T1W CNR & Phase CNR \\
\hline 0 & $8.3 \pm 1.4$ & $22.8 \pm 5.0$ & $2.0 \pm 1.0$ & $14.3 \pm 2.9$ & $3.2 \pm 1.2$ & $29.3 \pm 6.3$ \\
\hline 25 & $8.5 \pm 1.9(2.4 \%)$ & $22.9 \pm 2.7(0.4 \%)$ & $2.4 \pm 1.0(20.0 \%)$ & $14.5 \pm 1.6(1.4 \%)$ & $4.0 \pm 0.6(25.0 \%)$ & $35.5 \pm 4.1(21.2 \%)$ \\
\hline 75 & $8.8 \pm 1.8(6.0 \%)$ & $29.9 \pm 1.8(31.1 \%)$ & $4.1 \pm 1.0(105.0 \%)$ & $27.2 \pm 7.1(90.2 \%)$ & $4.9 \pm 0.3(53.0 \%)$ & $48.1 \pm 3.1(64.2 \%)$ \\
\hline 125 & $10.1 \pm 1.7(21.7 \%)$ & $39.4 \pm 0.8(72.8 \%)$ & $7.6 \pm 1.8(280.0 \%)$ & $41.2 \pm 4.8(188.1 \%)$ & $9.2 \pm 1.3(187.5 \%)$ & $62.7 \pm 2.6(114 \%)$ \\
\hline
\end{tabular}

a In between brackets, the percentage increase of contrast in respect to the contrast observed in absence of $\mathrm{Mn}^{2+}$ administration is shown.

Before $\mathrm{Mn}^{2+}$ infusion, phase imaging, thanks to its high CNR between and within gray and white matter, allowed the depiction of structures which are only discernible after $\mathrm{Mn}^{2+}$ infusion in $\mathrm{T}_{1}$-weighted imaging (see Figs. 2 and 3). Furthermore, other tissue structures such as white matter fiber tracts (fornix and mamillothalamic tract) not detectable on $\mathrm{T}_{1}$-weighted images both before and after $\mathrm{Mn}^{2+}$ enhancement were clearly discernible in phase images. Overall, by further increasing phase CNR which was, before $\mathrm{Mn}^{2+}$ infusion, higher than in the $\mathrm{T}_{1^{-}}$ weighted imaging, $\mathrm{Mn}^{2+}$ infusion enabled to further explore the brain structure and give additional information in comparison to the used $\mathrm{T}_{1}$-weighted imaging protocol. As such, $\mathrm{Mn}^{2+}$ can be considered as a MR phase contrast agent used to enhance neuro-architectonic contrast.

Twenty-four hours following $\mathrm{Mn}^{2+}$ administration, veinous structures were more pronounced, indicating an increase of susceptibility present in the veins that could be either due to an increase in the deoxyhemoglobin or manganese concentration. Independently of the origin of such vessel susceptibility changes, it has been shown that point-like susceptibility inclusions inside the vasculature contribute only to a very small extent to the phase contrast between gray and white matter $(13,31)$. Its contribution can be ruled out making the observed increase of contrast in tissue to be associated to the $\mathrm{Mn}^{2+}$ uptake in those regions.

The sensitivity of phase-based $\mathrm{Mn}^{2+}$ enhancement at 14.1T was also studied at different $\mathrm{Mn}^{2+}$ doses and compared with $\mathrm{T}_{1}$-weighted imaging in different brain ROIs. Qualitatively in Figures 3 and 4, contrast in regions rich or with active voltage gated calcium channels such as the dentate gyrus, cortex, and olfactory bulb continued to increase with the increasing $\mathrm{Mn}^{2+}$ doses for both imaging methods offering a better delineation of the cytoarchitectural features. Quantification of $\mathrm{R}_{1}$ changes from the $T_{1}$ maps, in the selected brain regions cited above, indicated a heterogeneous $\mathrm{R}_{1}$ increase $/ \mathrm{Mn}^{2+}$ uptake throughout the $\mathrm{Mn}^{2+}$ doses with the cortex exhibiting the smallest increase and the olfactory bulb and dentate gyrus the strongest increase (Fig. 5a and Table 1). These results are in agreement with a previous study made by Lee et al (9). The very low, but constant, $R_{1}$ increase observed in the corpus callosum $\left(+0.02 \mathrm{~s}^{-1} \pm\right.$ 0.03 from 0 to $125 \mathrm{mg} / \mathrm{kg}$ ) is a confirmation of the very weak presence of voltage gated calcium channels in white matter (32). $\mathrm{Mn}^{2+}$ distribution by means of the cer- ebrospinal fluid and paravascular spaces following systemic administration are the main pathways of $\mathrm{Mn}^{2+}$ enhancement in the rat brain (9). Nonspecific diffusion of $\mathrm{Mn}^{2+}$ ions might have an influence on the minimal $\mathrm{R}_{1}$ increase seen in the corpus throughout the $\mathrm{Mn}^{2+}$ doses. Alternatively, it could also be a consequence of the relatively low resolution of the $T_{1}$ maps and the point spread function due to the EPI readout, making the increase in $\mathrm{R}_{1}$ observed in the WM simply a partial volume effect. Nevertheless, the corpus callosum $R_{1}$ increase was very weak (when compared with the remaining regions) and could be considered non significant for the CNR study. From 0 to $125 \mathrm{mg} / \mathrm{kg}$, both $\Delta \mathrm{f}$ and $\Delta R_{1}$ increased monotonically in all regions despite correlation between $\Delta \mathrm{R}_{1}$ and $\Delta \mathrm{f}$ being different in the selected brain regions (8.8 in cortex, 5.4 in the DG and 13.1 in the $\mathrm{OB})$. Nonetheless, the increase in contrast observed in the $\Delta \mathrm{R}_{1}$ and $\Delta \mathrm{f}$ (see Fig. 5), when taking into account the phantom study results (Fig. 1), suggest $\mathrm{Mn}^{2+}$ enrichments are of the same order of magnitude, further supporting a close association between $R_{1}$, frequency shift increase and $\mathrm{Mn}^{2+}$ uptake. For the maximum $\mathrm{Mn}^{2+}$ dosage $(125 \mathrm{mg} / \mathrm{Kg})$, assuming relaxivity values hold in vivo, the following enrichments can be calculated: $21 \mu \mathrm{M}$ in the Cortex, $63 \mu \mathrm{M}$ in the DG, and $37 \mu \mathrm{M}$ in the OB.

The evaluation of the CNR increase following $\mathrm{Mn}^{2+}$ administration indicated that, overall, from 0 to $125 \mathrm{mg} /$ $\mathrm{kg}, \mathrm{Mn}^{2+}$ enhancement was more pronounced in phase imaging than in $\mathrm{T}_{1}$-weighted imaging protocol used although its fractional increase was greater for the latter (see Table 1). This observation is highly dependent on the sequence and parameters used to obtain the $\mathrm{T}_{1}$ weighted images. In this study, a gradient echo with a fixed TR of 1 second was applied to avoid respiration induced artifacts, under such constraints the optimal flip angle to obtain $\mathrm{T}_{1}$ contrast is $\sim 90^{\circ}$. Had our setup allowed for a three-dimensional acquisition, a TR as short as $10 \mathrm{~ms}$ could have been used together with an excitation of $11^{\circ}$ which would have increased our contrast per unit of time to the $\Delta \mathrm{R}_{1}$ by $\sim 10 \%$. Other studies in literature have used spin echo sequences $(9,10)$ or inversion recovery sequences (4). The use of a gradient echo with a short echo time was preferred to a spin echo sequence due to the $B_{1}$ inhomogeneity associated with the use of a surface coil. The signal from a spin echo sequence has a $\sin ^{3}\left(90^{*} B_{1}\right)$ dependence on $B_{1}$ (33), significantly limiting the range over which acceptable SNR 
and $\mathrm{T}_{1}$-weighting would be achieved on our setup and which can only be overcome by the usage of adiabatic refocusing pulses (34). A gradient echo sequence introduces a $\mathrm{Mn}^{2+}$ related signal attenuation due to $\mathrm{T}_{2}$ * decay. The reduction in contrast due to the use of a gradient echo sequence (given an apparent transverse relaxivity of $\mathrm{Mn}^{2+}$ ) is bound to be smaller than $50 \%$ for the largest $\mathrm{Mn}^{2+}$ dose, not enough to justify the 4-7 times reduction in $\mathrm{T}_{1}$-weighted contrast in respect to phase found in Table 1. Although it has been demonstrated inversion recovery-based sequences (such as the MPRAGE) offer superior $\mathrm{T}_{1}$-contrast with reduced sensitivity to $B_{1}$ homogeneity thanks to the use of adiabatic inversion pulses and efficient low flip angle excitations for the readout, analytical analysis of the contrast efficiency (35) of the spoiled gradient echo used in this study versus an MPRAGE sequence with the same echo time, constraint of 128 phase encoding steps per excitation (TR $=2-4 \mathrm{~s}$, flip angle $=8 \mathrm{TI}=1.5 \mathrm{~s}$ ) showed that the MPRAGE would not necessarily provide an improved $\mathrm{T}_{1}$-weighted contrast in comparison to our protocol when considering the typical relaxation parameters measured in the dentate gyrus $\left(\mathrm{R}_{1}\right.$ of $0.46 \mathrm{~s}^{-1}$ and a $\Delta \mathrm{R}_{1}$ of $0.35 \mathrm{~s}^{-1}$ following $\mathrm{Mn}^{2+}$ systemic administration at maximum dose of $125 \mathrm{mg} / \mathrm{kg}$ ). It is important to point out that, as the magnetic susceptibility induced frequency shift is linearly dependent to the static magnetic field, at lower magnetic fields (such as the typically available for animal scanning 4.7-7T), the CNR of the phase contrast will be lower and it remains to be determined at which field strength an optimized $\mathrm{T}_{1}$-weighted imaging protocol is likely to outperform phase imaging.

One of the pitfalls of in vivo phase imaging is its sensitivity to physiology and respiration induced artifacts due to the long echo time used. In our study, such artifacts were minimized by using respiration triggering. Another important limitation of phase imaging is the difficulty of obtaining reliable values in regions close to air/tissues interfaces such as the olfactory bulb. Despite the care taken when performing background filtering, the phase image in the olfactory bulb is pronouncedly affected by phase variations arising from air/tissue interfaces making structures situated at the extremities (such as the olfactory glomeruli, the glomerular layer) difficult to discern.

Phase-based MEMRI can, potentially, offer a higher resolution and contrast than that obtainable with $\mathrm{T}_{1}$ weighted imaging and be equally quantitative. To make phase-based $\mathrm{Mn}^{2+}$ quantification, it would be important to perform a quantitative susceptibility mapping $(36,37)$. The lack of this step on our analysis is likely to explain the difference in ordering observed between the frequency shift enhancement in the olfactory bulb, hippocampus, and in $\mathrm{R}_{1}$ maps. The measured frequency shift reflects not only the local increase of the $\mathrm{Mn}^{2+}$ uptake, but also its geometrical shape in respect to the static magnetic field. To be able to compute susceptibility maps, it would be beneficial to obtain a closer to isotropic resolution and bigger coverage than what was achievable with a surface coil. Two of the main difficulties when evaluating susceptibility maps is the fact that the forward model (role of macromolecular contribution (15), anisotropic susceptibility (38), and microstructure
(14)) is not fully understood and that the susceptibility values should be always seen as relative measure. In the case of studying $\mathrm{Mn}^{2+}$ uptake, $\mathrm{Mn}^{2+}$ can be considered as simple isotropic susceptibility contrast agent while any other bias of the QSM method will be the same prior and post to $\mathrm{Mn}^{2+}$ infusion. Also, from the results observed in this study using $R_{1}$ mapping (see Fig. 5a), white matter structures would form an ideal reference throughout the enrichment. Such an approach would offer fully quantitative $\mathrm{Mn}^{2+}$ uptake maps.

\section{CONCLUSIONS}

We conclude from the present study that MEMRI can be assessed by phase imaging at high magnetic field. Although $\mathrm{Mn}^{2+}$ had a stronger effect on $\mathrm{T}_{1}$-weighted imaging, phase imaging demonstrated to have superior CNR following $\mathrm{Mn}^{2+}$ systemic administration. Future work will be directed toward exploring high resolution and high contrast manganese enhanced phase images to obtain quantitative manganese uptake information by using quantitative susceptibility mapping.

\section{ACKNOWLEDGMENTS}

We thank Dr. Arthur Magill for the assistance given to the RF hardware. Supported in part by the EPFL-Merck Serono alliance, this study was supported by the Centre d'imagerie biomédicale of the UNIL, EPFL, UNIGE, CHUV and HUG as well as the Jeantet and Leenaards foundations.

\section{APPENDIX 1}

For a given echo time and as $\left[\mathrm{Mn}^{2+}\right]$ increases, the increased magnetic susceptibility induces an increase in phase but also a reduction in gradient echo (GRE) signal intensity due to $\mathrm{T}_{2}{ }^{*}$ attenuation. As phase CNR is correlated to both magnitude SNR and phase difference for a given echo time, MEMRI assessment by phase imaging at ultra-high field could be hampered by high $\left[\mathrm{Mn}^{2+}\right]$ if the reduction in $\mathrm{T}_{2}{ }^{*}$ is large, relative to the echo time used. To address this point, simulations were performed using a range of $\mathrm{Mn}^{2+}$ concentrations going beyond those found in the in vivo rodent brain following $\mathrm{Mn}^{2+}$ systemic administration (30). The GRE signal was simulated using the relaxation parameters experimentally found for the Dentate Gyrus $\left(\mathrm{R}_{1 \text { control }}, \Delta \mathrm{R}_{1}\right.$ at maximum dose of $125 \mathrm{mg} / \mathrm{kg}$ ) and $\mathrm{Mn}^{2+}$ relaxivities (reported in Section Relaxivity $r_{1}$ and $\delta f_{p m M}$ of $\mathrm{Mn}^{2+}$ at 14.1T). The GRE magnitude CNR was defined as the signal difference prior and post $\mathrm{Mn}^{2+}$ infusion (125 mg/kg). Phase CNR was defined similarly and as described in Eq. [3].

Figure A1 (a, b) shows the expected magnitude CNR for a 90 degree and Ernst angle setting respectively. As expected, in both cases the maximum GRE CNR is at $\mathrm{TE}=0$. However, due to the $\mathrm{T}_{2}{ }^{*}$ decay, a very fast CNR decrease is observed for the Ernst angle setting resulting in a decrease of magnitude signal as the echo time increases for high $\mathrm{Mn}^{2+}$ concentration. However, this decrease in signal intensity does not suggest a reduction of the phase CNR as it can be observed in Figure A1c. 

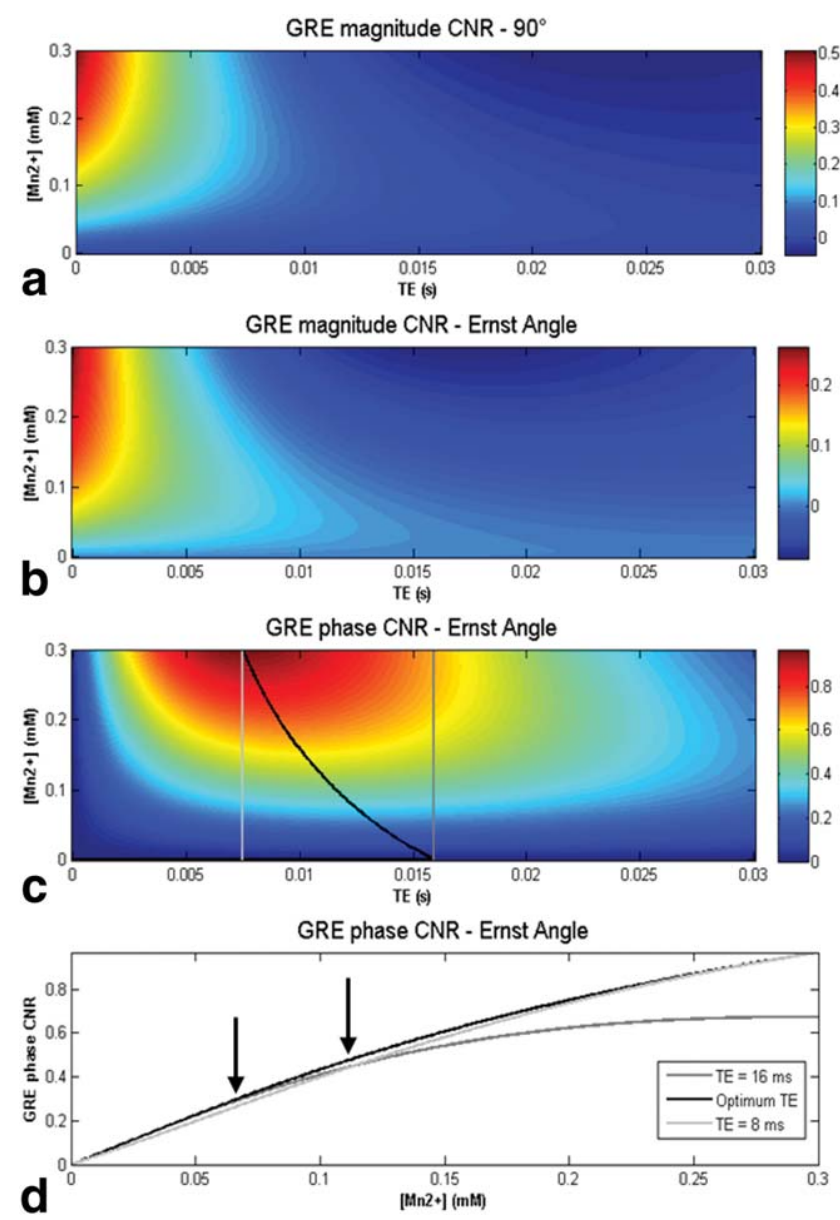

FIG. A1. a,b: Magnitude and (c) phase contrast to noise ratio of the GRE signal associated with the dentate gyrus as function of the $\mathrm{Mn}^{2+}$ concentration and echo time at $90^{\circ}$ (a) and at the Ernst angle $(b-d)$. In Figure A1.c, the black line shows the optimum echo time for a given $\mathrm{Mn}^{2+}$ concentration. The dark and light gray lines show the optimum echo time respectively at control and post $\mathrm{Mn}^{2+}$ infusion for the highest concentration. The plot in (d) shows the evolution of the phase CNR for these three different echo times. Black arrows point to the maximum $\mathrm{Mn}^{2+}$ concentration obtained in our study (0.064 $\mathrm{mM}$ for the Dentate Gyrus) and reported in literature [0.11 $\mathrm{mM}$ in Olfactory Bulb (30)].

Nevertheless it suggests that the optimum echo time should be reduced for higher $\mathrm{Mn}^{2+}$ concentrations (as shown by the black line). The optimum echo time decreases from $16 \mathrm{~ms}$ to $8 \mathrm{~ms}\left(\right.$ at $\left[\mathrm{Mn}^{2+}\right]=0.3 \mathrm{mM}$ ) due to the $\mathrm{T}_{2}{ }^{*}$ decay. The plot in Figure A1d shows that, if the optimum echo at pre- $\mathrm{Mn}^{2+}$ infusion is indeed kept constant, the phase CNR starts to saturate at high concentrations of $\mathrm{Mn}^{2+}$ (as shown by the dark gray line) although the frequency difference would continue to increase linearly.

Consequently, the use of a shorter echo time for post $\mathrm{Mn}^{2+}$ enrichment would have been preferable as it can be observed at TE $=8 \mathrm{~ms}$ where the phase CNR evolution is close to optimum (light gray line). However, when taking into account the range of $\mathrm{Mn}^{2+}$ concentration obtained in the study ( 0 to $0.064 \mathrm{mM}$ ), at $\mathrm{TE}=16$ ms no saturation effect is observed as the phase CNR increased linearly with the increased tissue $\left[\mathrm{Mn}^{2+}\right]$.

\section{REFERENCES}

1. Chandra SV, Shukla GS. Role of iron deficiency in inducing susceptibility to manganese toxicity. Arch Toxicol 1976;35:319-323.

2. Wolf GL, Baum L. Cardiovascular toxicity and tissue proton T1 response to manganese injection in the dog and rabbit. AJR Am J Roentgenol 1983;141:193-197.

3. Jankovic J. Searching for a relationship between manganese and welding and Parkinson's disease. Neurology 2005;64:2021-2028.

4. Tucciarone J, Chuang KH, Dodd SJ, Silva A, Pelled G, Koretsky AP. Layer specific tracing of corticocortical and thalamocortical connectivity in the rodent using manganese enhanced MRI. Neuroimage 2009;44:923-931.

5. Yu X, Wadghiri YZ, Sanes DH, Turnbull DH. In vivo auditory brain mapping in mice with Mn-enhanced MRI. Nat Neurosci 2005;8:961968.

6. Aoki I, Wu YJ, Silva AC, Lynch RM, Koretsky AP. In vivo detection of neuroarchitecture in the rodent brain using manganese-enhanced MRI. Neuroimage 2004;22:1046-1059.

7. Silva AC, Lee JH, Wu CW, Tucciarone J, Pelled G, Aoki I, Koretsky AP. Detection of cortical laminar architecture using manganeseenhanced MRI. J Neurosci Methods 2008;167:246-257.

8. Morita H, Ogino T, Seo Y, Fujiki N, Tanaka K, Takamata A, Nakamura S, Murakami M. Detection of hypothalamic activation by manganese ion contrasted T(1)-weighted magnetic resonance imaging in rats. Neurosci Lett 2002;326:101-104.

9. Lee JH, Silva AC, Merkle H, Koretsky AP. Manganese-enhanced magnetic resonance imaging of mouse brain after systemic administration of $\mathrm{MnCl} 2$ : dose-dependent and temporal evolution of $\mathrm{T} 1$ contrast. Magn Reson Med 2005;53:640-648.

10. Chuang KH, Belluscio L, Koretsky AP. In vivo detection of individual glomeruli in the rodent olfactory bulb using manganese enhanced MRI. Neuroimage 2010;49:1350-1356.

11. Duyn JH, van Gelderen P, Li TQ, de Zwart JA, Koretsky AP, Fukunaga M. High-field MRI of brain cortical substructure based on signal phase. Proc Natl Acad Sci U S A 2007;104:11796-11801.

12. Rauscher A, Sedlacik J, Barth M, Mentzel HJ, Reichenbach JR. Magnetic susceptibility-weighted MR phase imaging of the human brain. AJNR Am J Neuroradiol 2005;26:736-742.

13. Marques JP, Maddage R, Mlynarik V, Gruetter R. On the origin of the MR image phase contrast: an in vivo MR microscopy study of the rat brain at $14.1 \mathrm{~T}$. Neuroimage 2009;46:345-352.

14. He X, Yablonskiy DA. Biophysical mechanisms of phase contrast in gradient echo MRI. Proc Natl Acad Sci U S A 2009;106:13558-13563.

15. Zhong K, Leupold J, von Elverfeldt D, Speck O. The molecular basis for gray and white matter contrast in phase imaging. Neuroimage 2008;40:1561-1566.

16. Fukunaga M, Li TQ, van Gelderen P, et al. Layer-specific variation of iron content in cerebral cortex as a source of MRI contrast. Proc Natl Acad Sci U S A 2010;107:3834-3839.

17. Schweser F, Deistung A, Lehr BW, Reichenbach JR. Quantitative imaging of intrinsic magnetic tissue properties using MRI signal phase: an approach to in vivo brain iron metabolism? Neuroimage 2011;54:2789-2807.

18. Lodygensky GA, Marques JP, Maddage R, Perroud E, Sizonenko SV, Huppi PS, Gruetter R. In vivo assessment of myelination by phase imaging at high magnetic field. Neuroimage 2012;59:1979-1987.

19. Liu C, Li W, Johnson GA, Wu B. High-field (9.4 T) MRI of brain dysmyelination by quantitative mapping of magnetic susceptibility. Neuroimage 2011;56:930-938.

20. Lee J, Shmueli K, Kang BT, Yao B, Fukunaga M, van Gelderen P, Palumbo S, Bosetti F, Silva AC, Duyn JH. The contribution of myelin to magnetic susceptibility-weighted contrasts in high-field MRI of the brain. Neuroimage 2012;59:3967-3975.

21. Gruetter R. Automatic, localized in vivo adjustment of all first- and second-order shim coils. Magn Reson Med 1993;29:804-811.

22. Gowland P, Mansfield P. Accurate measurement of T1 in vivo in less than 3 seconds using echo-planar imaging. Magn Reson Med 1993;30: 351-354.

23. Chuang KH, Koretsky A. Improved neuronal tract tracing using manganese enhanced magnetic resonance imaging with fast $\mathrm{T}(1)$ mapping. Magn Reson Med 2006;55:604-611.

24. van der Zwaag W, Marques JP, Lei H, Just N, Kober T, Gruetter R. Minimization of Nyquist ghosting for echo-planar imaging at ultra- 
high fields based on a "negative readout gradient" strategy. J Magn Reson Imaging 2009;30:1171-1178.

25. Nelder JA, Mead R. A Simplex-Method for Function Minimization. Comput J 1965;7:308-313.

26. Goldstein RM, Zebker HA, Werner CL. Satellite radar interferometry two-dimensional phase unwrapping. Radio Sci 1988;23:713-720.

27. Marques JP, Bowtell RW. Using forward calculations of the magnetic field perturbation due to a realistic vascular model to explore the BOLD effect. NMR Biomed 2008;21:553-565.

28. Kukley M, Capetillo-Zarate E, Dietrich D. Vesicular glutamate release from axons in white matter. Nat Neurosci 2007;10:311-320.

29. Silva AC, Lee JH, Aoki I, Koretsky AP. Manganese-enhanced magnetic resonance imaging (MEMRI): methodological and practical considerations. NMR Biomed 2004;17:532-543.

30. Chuang KH, Koretsky AP, Sotak CH. Temporal changes in the T1 and $\mathrm{T} 2$ relaxation rates (DeltaR1 and DeltaR2) in the rat brain are consistent with the tissue-clearance rates of elemental manganese. Magn Reson Med 2009;61:1528-1532.

31. Lee J, Hirano Y, Fukunaga M, Silva AC, Duyn JH. On the contribution of deoxy-hemoglobin to MRI gray-white matter phase contrast at high field. Neuroimage 2010;49:193-198.
32. Kelly KM, Ikonomovic MD, Abrahamson EE, Kharlamov EA, Hentosz TM, Armstrong DM. Alterations in hippocampal voltage-gated calcium channel alpha 1 subunit expression patterns after kainateinduced status epilepticus in aging rats. Epilepsy Res 2003;57:15-32.

33. Haacke EM. Magnetic resonance imaging: physical principles and sequence design. New York: Wiley; 1999. xxvii, $914 \mathrm{p.}$

34. van de Looij Y, Kunz N, Huppi P, Gruetter R, Sizonenko S. Diffusion tensor echo planar imaging using surface coil transceiver with a semiadiabatic RF pulse sequence at 14.1T. Magn Reson Med 2011;65: 732-737.

35. Marques JP, Kober T, Krueger G, van der Zwaag W, Van de Moortele PF, Gruetter R. MP2RAGE, a self bias-field corrected sequence for improved segmentation and T1-mapping at high field. Neuroimage 2010;49:1271-1281.

36. Wharton S, Bowtell R. Whole-brain susceptibility mapping at high field: a comparison of multiple- and single-orientation methods. Neuroimage 2010;53:515-525.

37. Shmueli K, de Zwart JA, van Gelderen P, Li TQ, Dodd SJ, Duyn JH. Magnetic susceptibility mapping of brain tissue in vivo using MRI phase data. Magn Reson Med 2009;62:1510-1522.

38. Liu C. Susceptibility tensor imaging. Magn Reson Med 2010;63:14711477. 